\title{
Bubble Clouds: 3D Display Composed of Soap Bubble Cluster
}

\author{
Yuki Kubo $^{(\bowtie)}{ }^{(}$, Hirobumi Tomita ${ }^{1}$, Shuta Nakamae ${ }^{1}$, \\ Takayuki Hoshi ${ }^{2}$, and Yoichi Ochiai ${ }^{3}$ \\ 1 University of Tsukuba, 1-1-1 Tennodai, Tsukuba, Ibaraki 305-8573, Japan \\ \{kubo, tomita, nakamae\}@iplab.cs.tsukuba.ac.jp \\ 2 The University of Tokyo, 7-3-1 Hongo, Bunkyo-ku, Tokyo 113-8658, Japan \\ star@star.rcast.u-tokyo.ac.jp \\ 3 University of Tsukuba, 1-2 Kasuga, Tsukuba, Ibaraki 305-8550, Japan \\ wizard@slis.tsukuba.ac.jp
}

\begin{abstract}
We examine Bubble Clouds composed of a bubble cluster for a threedimensional display. A bubble cluster is flexible in that its shape can be modified and it can float in the air with helium gas confinement of the bubbles. By varying the density of the bubble clusters, we project images onto the bubbles without requiring fog confinement or use of special equipment. Moreover, we investigate whether a soap bubble cluster can become interactive by electrifying it.
\end{abstract}

Keywords: Bubble display $\cdot$ Soap bubbles $\cdot$ Ephemeral user interfaces

\section{Introduction}

Previously, bubble displays have been achieved as singular [9], multi-layered [10], or multiple bubble surfaces [6]. A single bubble surface cannot display three-dimensional (3D) structures. Although a multi-layered bubble surface can show a semblance of 3D assemblies, they cannot display a complete range of the same.

Bubble displays have several limitations in their size, structure, and projection methods. This motivated us to use a soap bubble cluster as a display system (Fig. 1). We can express 3D objects by reshaping the bubble clusters without fog confinement, and can project images onto the bubbles. In this study, we present Bubble Clouds [5], as displays that use a bubble cluster (Fig. 1). A bubble cluster is flexible in that its shape can be modified. In this regard, we examined five shapes of a bubble cluster. Furthermore, it can float in the air by helium gas confinement of the bubbles and adjustment of the bubble density. We can project images on the bubble clouds without requiring fog confinement or special equipment, and by confining helium in the bubbles. We can provide the utility of bubble clusters when producing a bubble display. For example, we can project images onto a bubble cluster without fog confinement, as shown in Fig. 2.

\section{Related Work}

Research on bubble cluster displays is related to studies that involve bubble displays and ephemeral user interfaces. 


\subsection{Bubble Display}

Some research studies have proposed displaying images by using soap bubbles. For example, Bubble Cosmos [9] provides tangible interactions with bubbles, and a sound is played when a user bursts a bubble. Bubble Cosmos is a single-bubble display that projects an image onto a bubble with fog confinement. FRAGWRAP [7] encapsulates fragrances in a bubble confined with fog, so that when the bubble bursts, the fragrance is released to the user. SensaBubble [13] is a display system that uses the fog confinement of the bubbles to deliver information to users by using a projector and fragrances. Colloidal Displays $[10,11]$ project images onto a soap film by using the ultrasound waves emitted from an ultrasonic-phased array. The reflectance can be varied by vibrating the film using the phased array. Similar to this work, we also used an ultrasonic-phased array as one of the methods to project images on a bubble cluster. Liquids, Smoke, and Soap Bubbles [14] form bubble display that consists of bubbles, a soap liquid, and fog, and can be made to interact by moving or blowing bubbles over a dark surface. Sahoo et al. [12] proposed a method that could alter the trajectory of a bubble by confining an electrostatically charged fog in the bubble and applying an electric field. In comparison with these displays that utilize single soap bubbles or a soap film, our proposed Bubble Clouds utilize a bubble cluster.

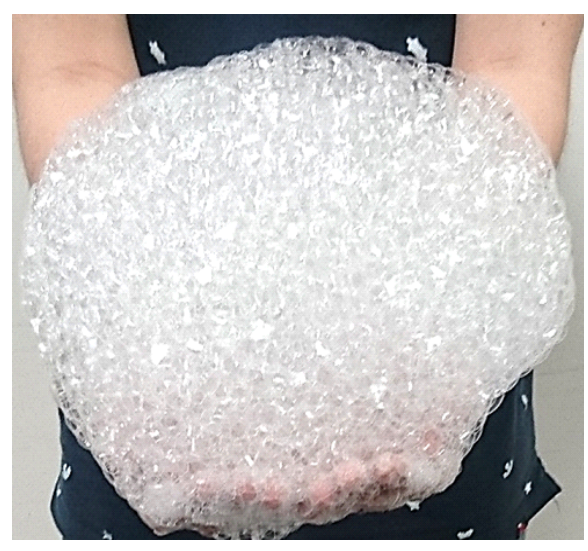

Fig. 1. A bubble cluster.

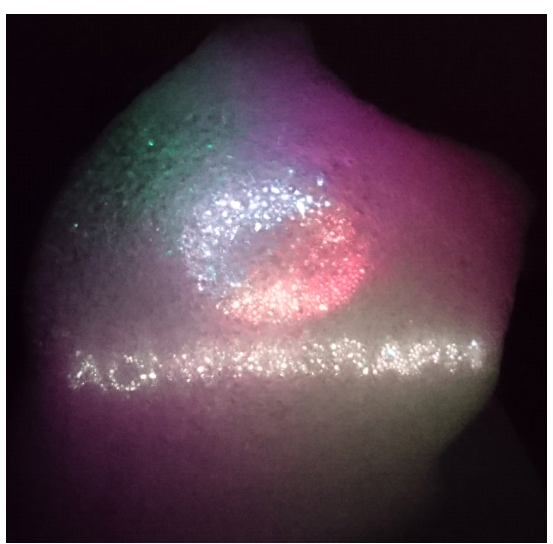

Fig. 2. Dense Bubble Cloud.

An example of a display that uses multiple soap bubbles is Shaboned Display [4]. This display consists of an array of soap bubbles, and each soap bubble works as a pixel of an image. Flogos [3] is a device that can form characters and logos that can float by utilizing soap clusters. Bubble Clouds can combine multiple bubbles into a single bubble cluster, and can express 3D objects by reshaping the bubble cluster. Furthermore, Bubble Clouds can project images onto the bubble cluster. 


\subsection{Ephemeral User Interfaces}

A soap bubble display is a type of an ephemeral user interface [2]. Similarly, several displays using ephemeral materials such as water, smoke, and fog have been previously proposed. For example, Barnum et al. [1] proposed a display that projected images on multi-layered water drops. Cloud Display [16] is a space filling display composed of smoke rings. Tangible Sound [18] uses fluid water as an input of a musical instrument. By adjusting the flowing water, a sound is produced. HydroMorph [8] is a water display that can vary the shape by sensing the users input by camera. Cool Interaction with Calm Technologies [17] is a multi-touch screen built from an ice-wall, that can detect the palm of a user and achieve multi-touch by using an infrared camera. Cloud Interface [15] is a mid-air display that can move in the air. It consists of a blimp, a gondola, and a projector. Bubble Clouds use soap bubbles as the ephemeral material.

\section{Implementation}

We use a bubble generator to produce the bubbles that comprise the bubble cluster (Fig. 3). The bubble generator consists of a helium cylinder, a pressure gage, an air tube, a bucket containing soap solution, and an acrylic case with five holes. To generate bubbles that can float, helium gas is passed through the soap solution that passes through the holes in the acrylic case. A floatable bubble cluster is generated by separating the bubbles from the bucket by using an air tube having an inner diameter of $3 \mathrm{~mm}$. The pressure gage regulates the pressure inside the air tube. We place an acrylic panel on the bucket with a $25 \mathrm{~cm}$ square hole at its center from which the bubbles escape from the bucket. To hold the soap bubble solution and store bubbles, we use a bucket with a diameter of $45 \mathrm{~cm}$ and height of $15 \mathrm{~cm}$. The solution should generate bubbles that do not burst easily, and we use a solution mixture of water, a detergent that includes a surfactant, and laundry starch that includes polyvinyl alcohol in a ratio of 5:1:5.

An ultrasonic-phased array with 283 ultrasonic transducers is shown in Fig. 4. Ultrasound waves, generated from an ultrasonic-phased array and emitted to the bubbles, change the reflectance of the soap film, and thereby produce vibrations and project images onto the film. An ultrasonic-phased array generates an ultrasonic wave front to stimulate the entire surface of the bubble cluster.

\section{Bubble Clouds}

\subsection{Dense Bubble Cloud}

When the bubbles are small, they seem to appear as if they are pixels of a projected image, as shown in Fig. 2. Dense Bubble Cloud allows each bubble to function as an individual pixel, and hence does not require fog confinement or additional material to project images. 


\subsection{Sparse Bubble Cloud}

Images cannot be projected onto bubbles when their diameter is extremely large because the soap film permeates light. However, we can project images onto such bubbles by using ultrasonic waves, and we call this Sparse Bubble Cloud.

In Colloidal Display, a method of projecting images by changing the reflection properties of the film by ultrasonic wave was introduced. We investigated whether this method can work effectively for bubble clusters. Fig. 5a shows a state when the film is not vibrating. The image is not projected by light; it is permeated. Fig. 5b depicts the bubble cluster when the reflectance is varied by applying an ultrasonic wave to the soap film via an ultrasonic-phased array. Compared with Fig. 5a, the reflectance changes in Fig. 5b. We attempted to project images onto a bubble cluster by using an ultrasonicphased array (Fig. 4). The image projected was slightly clear compared with a bubble cluster without ultrasonic waves (Fig. 6).

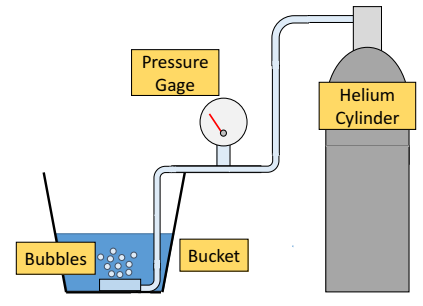

Fig. 3. Bubble generator.

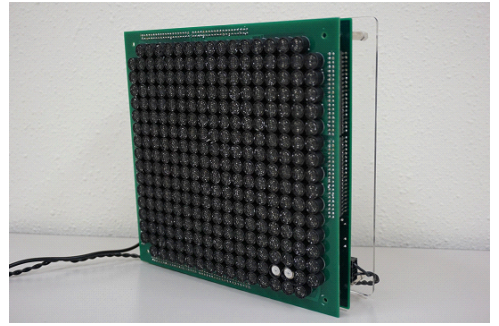

Fig. 4. Ultrasonic-phased array.
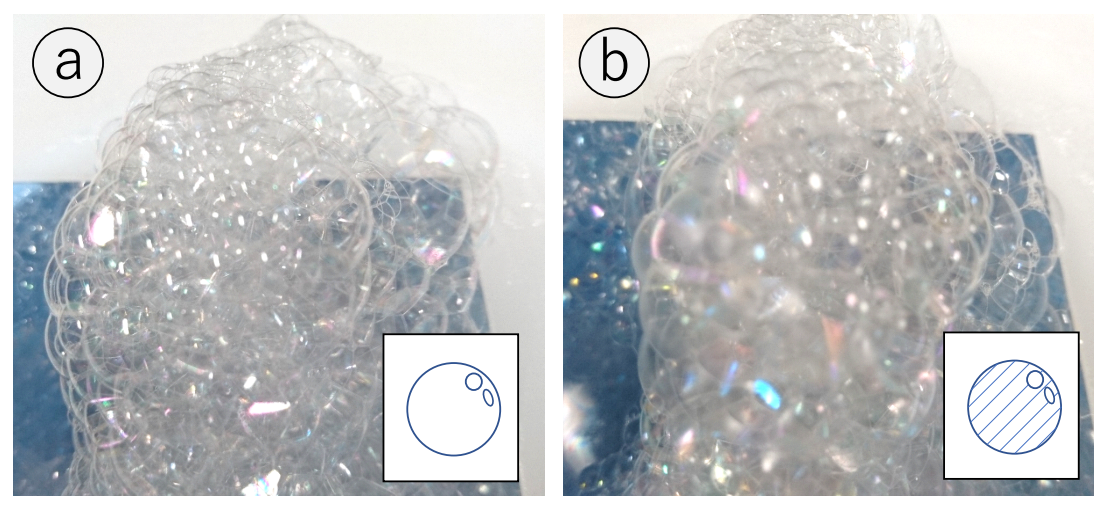

Fig. 5. Variation of the reflectance of a bubble by controlling ultrasound waves. (a) Non-vibrating soap film. (b) Vibrating soap film. 


\section{Evaluation of Bubble Generator}

We evaluated the effect of the pressure and diameter of the holes of the acrylic case. We conducted this experiment to evaluate whether these conditions meet the smallest diameter requirement of the bubbles that enables the display to float. The pressure was measured by using a pressure gage. In our experiment, we applied the following values: $0.02,0.04$, and $0.06 \mathrm{MPa}$. We consider two diameter sizes for the acrylic cases: 0.02 and $0.04 \mathrm{~mm}$, and there are five holes in each case, positioned at the same distance from each other (Fig. 7). The diameter of a bubble cluster is measured by a ruler. The bubbles cannot be uniform in size, and thus, we measured the diameters of numerous bubbles and averaged the result.
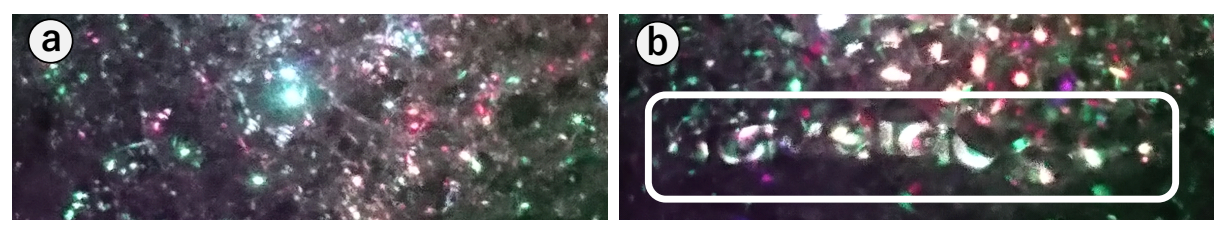

Fig. 6. Sparse Bubble Cloud using an ultrasonic-phased array: (a) non-vibrating bubbles with projection, and (b) vibrating bubbles with projection (projection characters:"ACM SIGGRAPH").

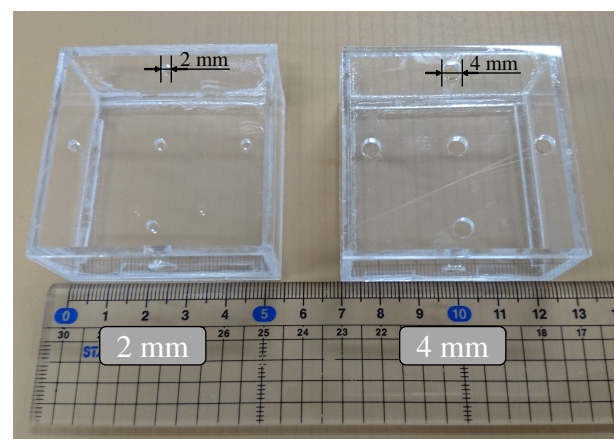

Fig. 7. Acrylic cases with five holes (scale is $54 \times 54 \times 24 \mathrm{~mm}$ ). Left: hole diameter of $2 \mathrm{~mm}$. Right: hole diameter of $4 \mathrm{~mm}$.

The results are shown in Table 1. We observe that higher pressures or larger holes produce larger bubbles. These conditions satisfy the smallest diameter requirement enabling a bubble to float. We chose the following conditions to generate the smallest bubbles possible: pressure of $0.02 \mathrm{MPa}$ and diameter of $2.0 \mathrm{~mm}$. 


\section{Shape of Bubble Cluster}

We investigated the shapes that could be generated with a bubble cluster. In this experiment, we formed five shapes with the bubble cluster, namely, cube, quadrangular pyramid, sphere, mountain, and two mountains. Fig. 8 shows the different cluster shapes. Bubble cluster reshaping was performed by using a $5 \times 30 \mathrm{~cm}$ plate. We poured the soap bubble solution into a bucket and fixed an acrylic case to the bottom. A helium cylinder was connected to the acrylic case by an air tube. Helium gas was released at a constant rate from the acrylic case into the soap bubble solution. The reason helium gas was confined is because it is easy to process and maintain the shape of the bubble cluster without being influenced by gravity. Bubble clusters escaped from a $25 \times 25 \mathrm{~cm}$ hole on a plate that was placed on the bucket. Thus, the user could reshape the bubble cluster to any shape.

Table 1. Effect of hole diameter and pressure on the bubble diameter.

\begin{tabular}{|l|c|c|c|}
\hline Pressure (MPa) & 0.02 & 0.04 & 0.06 \\
\hline Holes Diameter $(\mathrm{mm})$ & $10 \mathrm{~mm}$ & $15 \mathrm{~mm}-20 \mathrm{~mm}$ & $20 \mathrm{~mm}-30 \mathrm{~mm}$ \\
\hline 2.0 & $15 \mathrm{~mm}-25 \mathrm{~mm}$ & $25 \mathrm{~mm}-30 \mathrm{~mm}$ & $30 \mathrm{~mm}-35 \mathrm{~mm}$ \\
\hline
\end{tabular}

Fig. 8 shows the five shapes of the bubble cluster we produced. The cube shown in Fig. 8a was formed by placing a cubic mold on the top of the hole. The cubic mold was made from five plates, and we separated each plate individually to create a cube. The quadrangular pyramid shown in Fig. 8b was formed by sharpening the tip by scraping the bubble cluster using a plate. The sphere and mountain shown in Fig. 8c and d, respectively were generated by adjusting the hole with the plates. The two mountains shown in Fig. 8e were formed by scraping the middle of the bubble cluster.

\section{Interaction with Electrified Bubble Cluster}

We investigated whether an electrified soap bubble cluster could be interactive. This was verified in an indoor still air environment. To electrify the bubble clusters, we used a polyvinyl chloride pipe and tissue paper. Static electricity was generated by their friction that was charged by touching the bubble cluster with the polyvinyl chloride pipe in the air. After that, we examined how the bubble cluster behaved when a user placed a hand near the bubble cluster. Consequently, we could observe a bubble cluster being tracked by the hand of a user. As shown in Fig. 9, the bubble cluster moves in the same direction as the hand. We believe that this occurs owing to the Coulomb force between the hand of a user and the bubble cluster. Therefore, we can manipulate the soap bubble cluster in mid-air. We also noticed that the tracking was lost once the Coulomb force ceased to exist. 


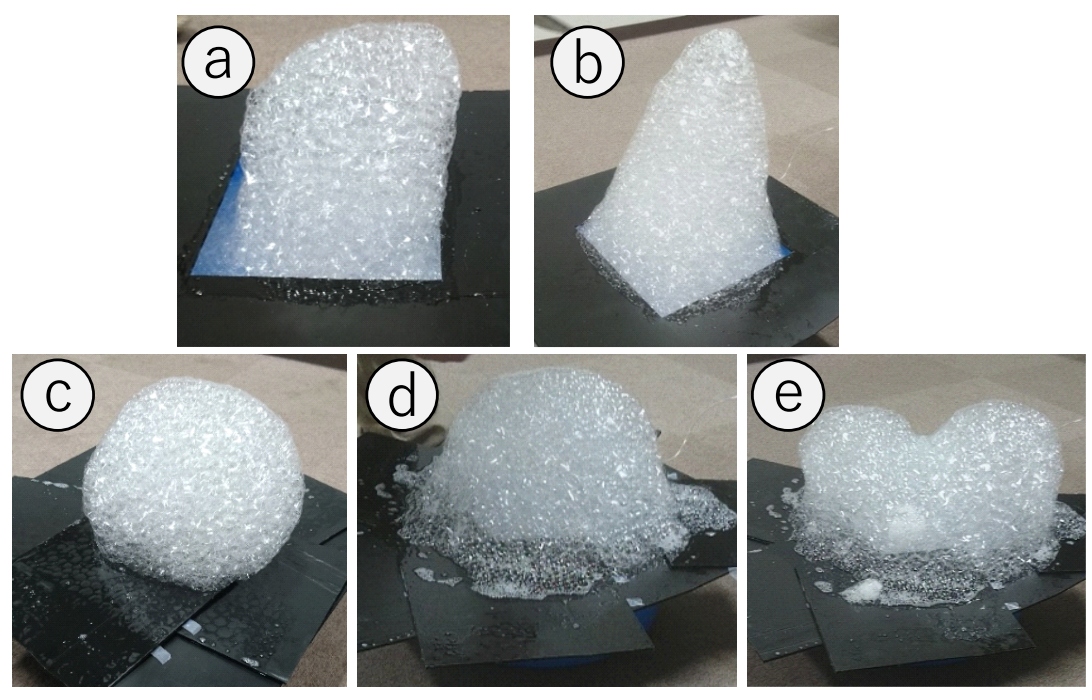

Fig. 8. Five shapes of bubble cluster: (a) cube, (b) quadrangular pyramid, (c) sphere, (d) mountain, and (e) two mountains.

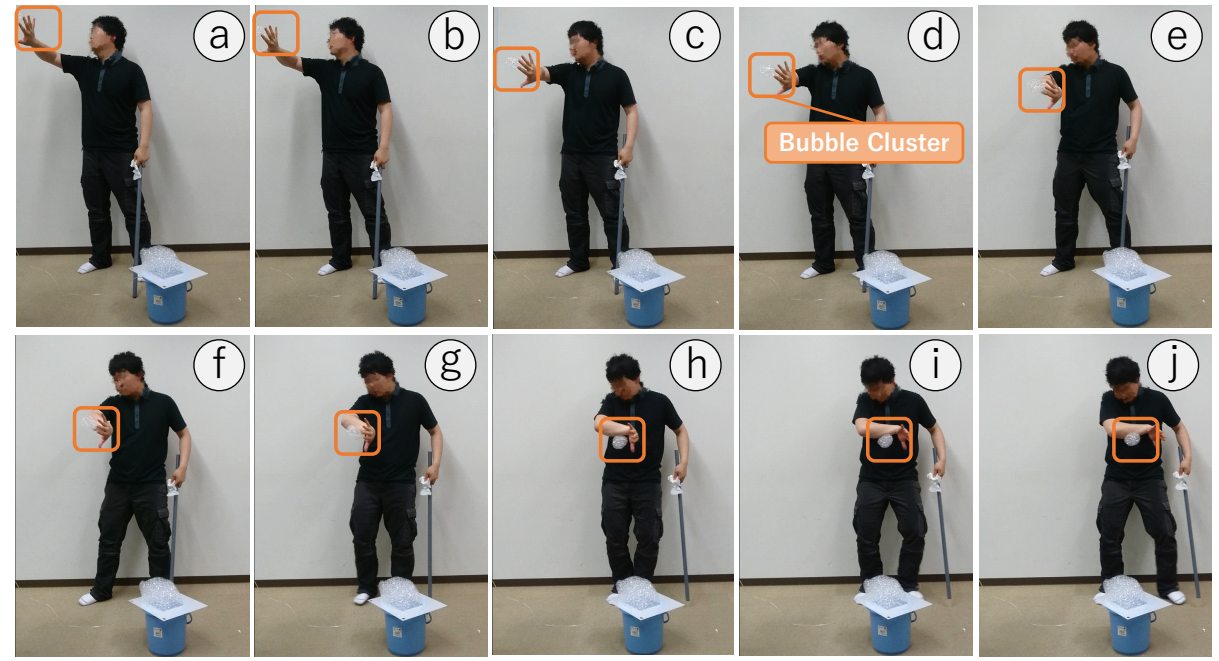

Fig. 9. Time-lapse of interaction between user and bubble cluster. 


\section{Example Application}

\subsection{Bubble Characters}

We propose that characters could be generated by using a Bubble Cloud. A user could then interact with the characters using his/her hands and with objects. For example, we could formulate a game in which a user could use a toy sword to attack the generated characters (e.g., monsters). Each character could be projected differently, and thus, a user would be able to classify each character.

\section{Discussion and Future Work}

We attempted to project images onto a Sparse Bubble Cloud in mid-air by using an ultrasonic-phased array. However, this Sparse Bubble Cloud moved because of the ultrasonic wave emitted from the phased array. This suggests that if we project images onto Sparse Bubble Cloud in mid-air, we must track the movement of the bubble cluster and counter the effect of the ultrasonic wave to prevent the movement.

In our experiment, we observed the phenomenon of a bubble cluster tracking hand of a user. However, it was unable to track when its size was extremely large. Our results showed that the Coulomb force between the hand of the user and bubble cluster was not sufficiently strong. It is to be noted that we electrified the bubble cluster by using a simple method in this experiment. For this reason, we believe that the bubble cluster could be moved if it was strongly electrified by a specific equipment. Moreover, this interaction was easily affected by wind.

Dense Bubble Cloud is a type of Bubble Cloud that could project an image to the surface of a bubble cluster. In addition, we found that we can utilize an entire soap bubble cluster including the bubbles inside, as pixels and use it as a 3D display (Fig. 10). This is possible apparently because each of the bubbles in the bubble cluster reflects and permeates light. In the future, to achieve 3D projection on a bubble cluster, we will investigate conditions such as the diameter of the bubbles that compose the bubble cluster. We will also examine if we can realize a true 3D display by using multiple planes because presently our display only has a single plane.

In this work, we used panels to reshape the bubble cluster. However, this method has a poor reproducibility in terms of shape because the process of reshaping is different for different users. The reproducibility of shape can be ensured by a method that uses an ultrasonic-phased array. In the future, we will reshape the bubble cluster automatically to ensure shape reproducibility by using an ultrasonic-phased array.

Furthermore, in the future, we plan to conduct quantitative evaluations such as how long the bubble cluster lasts, and character recognition rate. Additionally, we aim to conduct quantitative evaluation such as the user perception of the applicability of the display for entertainment purposes.

\section{Conclusion}

In this study, we presented Bubble Clouds using bubble cluster as displays. A bubble cluster is flexible because its shape can be varied and it can float in the air by helium 
confinement. We introduced two types of Bubble Clouds, namely, Dense Bubble Cloud and Sparse Bubble Cloud. By changing the density of the bubble cluster, images could be projected onto it without fog confinement or use any special equipment. We formed five shapes with the bubble clusters: cube, quadrangular pyramid, sphere, mountain, and two mountains. Moreover, we also explored whether an electrified soap bubble cluster could be interactive.

In the future, to realize 3D projection on a bubble cluster, we will investigate conditions such as the diameter of the bubbles that compose the bubble cluster. Furthermore, we plan to explore reshaping the bubble clusters automatically to ensure shape reproducibility by using an ultrasonic-phased array.
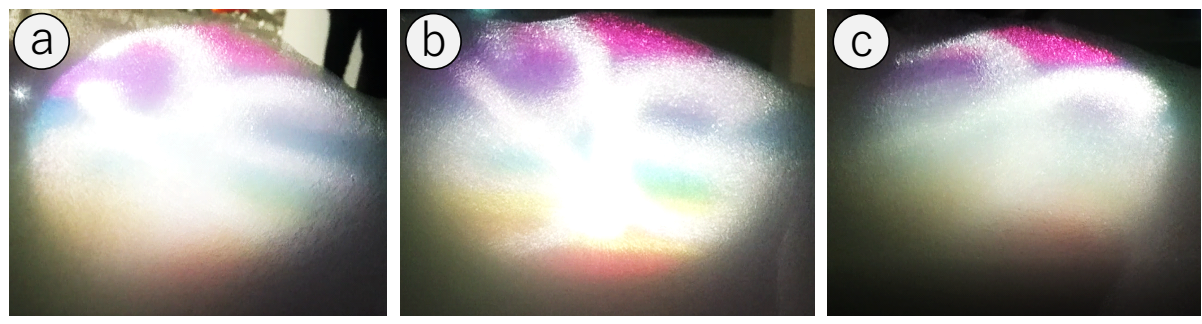

Fig. 10. 3D form display composed of bubble cluster. Photo from the (a) left, (b) front, (c) right (projecting the inverted ace2016 logo).

\section{References}

1. Barnum, P.C., Narasimhan, S.G., Kanade, T.: A multi-layered display with water drops. ACM Transactions on Graphics 29(4), 76:1-76:7 (July 2010), http://doi . acm. org/10. $1145 / 1778765.1778813$

2. Döring, T., Sylvester, A., Schmidt, A.: A design space for ephemeral user interfaces. In: Proceedings of the 7th International Conference on Tangible, Embedded and Embodied Interaction. pp. 75-82. TEI '13, ACM, New York, NY, USA (2013), http://doi . acm.org/ $10.1145 / 2460625.2460637$

3. Global special effects, I.: Flogos (2008), http://globalspecialeffects. com/flogos. aspx

4. Hirayama, S., Kakehi, Y.: Shaboned Display: An interactive substantial display using soap bubbles. In: ACM SIGGRAPH 2010 Emerging Technologies. SIGGRAPH '10, ACM (2010), http://doi .acm.org/10.1145/1836821.1836842

5. Kubo, Y., Tomita, H., Nakamae, S., Hoshi, T., Ochiai, Y.: Bubble Cloud: Projection of an image onto a bubble cluster. In: Proceedings of the 13th International Conference on Advances in Computer Entertainment Technology. pp. 41:1-41:4. ACE2016, ACM, New York, NY, USA (2016), http: //doi . acm.org/10.1145/3001773.3001815

6. Kwon, H., Jaiswal, S., Benford, S., Seah, S.A., Bennett, P., Koleva, B., Schnädelbach, H.: FugaciousFilm: Exploring attentive interaction with ephemeral material. In: Proceedings of the 33rd Annual ACM Conference on Human Factors in Computing Systems. pp. 1285-1294. 
CHI '15, ACM, New York, NY, USA (2015), http://doi . acm.org/10. 1145/2702123. 2702206

7. Kyono, Y., Yonezawa, T., Nozaki, H., Nakazawa, J., Tokuda, H.: FRAGWRAP: Fragranceencapsulated and projected soap bubble for scent mapping. In: Proceedings of the 2013 ACM Conference on Pervasive and Ubiquitous Computing Adjunct Publication. pp. 311-314. UbiComp '13 Adjunct, ACM (2013), http: //doi . acm.org/10.1145/2494091.2494187

8. Nakagaki, K., Totaro, P., Peraino, J., Shihipar, T., Akiyama, C., Shuang, Y., Ishii, H.: HydroMorph: Shape changing water membrane for display and interaction. In: Proceedings of the TEI '16: Tenth International Conference on Tangible, Embedded, and Embodied Interaction. pp. 512-517. TEI '16, ACM (2016), http://doi . acm.org/10. 1145/2839462 . 2856517

9. Nakamura, M., Inaba, G., Tamaoki, J., Shiratori, K., Hoshino, J.: Mounting and application of bubble display system: Bubble cosmos. In: Proceedings of the 2006 ACM SIGCHI International Conference on Advances in Computer Entertainment Technology. ACE '06, ACM (2006), http://doi . acm.org/10.1145/1178823.1178879

10. Ochiai, Y., Oyama, A., Hoshi, T., Rekimoto, J.: The Colloidal Metamorphosis: Time division multiplexing of the reflectance state. Computer Graphics and Applications, IEEE 34(4), 42$51(2014)$

11. Ochiai, Y., Oyama, A., Hoshi, T., Rekimoto, J.: Theory and Application of the Colloidal Display: Programmable bubble screen for computer entertainment. In: Proceeding of the 10th International Conference on Advances in Computer Entertainment - Volume 8253. pp. 198-214. ACE'13, Springer-Verlag (2013), http://dx.doi.org/10.1007/ 978-3-319-03161-3_14

12. Sahoo, D.R., Martinez Plasencia, D., Subramanian, S.: Control of non-solid diffusers by electrostatic charging. In: Proceedings of the 33rd Annual ACM Conference on Human Factors in Computing Systems. pp. 11-14. CHI '15, ACM, New York, NY, USA (2015), http://doi.acm.org/10.1145/2702123.2702363

13. Seah, S.A., Martinez Plasencia, D., Bennett, P.D., Karnik, A., Otrocol, V.S., Knibbe, J., Cockburn, A., Subramanian, S.: SensaBubble: A chrono-sensory mid-air display of sight and smell. In: Proceedings of the $32 \mathrm{Nd}$ Annual ACM Conference on Human Factors in Computing Systems. pp. 2863-2872. CHI '14, ACM, New York, NY, USA (2014), http: //doi.acm.org/10.1145/2556288.2557087

14. Sylvester, A., Döring, T., Schmidt, A.: Liquids, Smoke, and Soap Bubbles: Reflections on materials for ephemeral user interfaces. In: Proceedings of the Fourth International Conference on Tangible, Embedded, and Embodied Interaction. pp. 269-270. TEI '10, ACM (2010), http://doi .acm.org/10.1145/1709886.1709941

15. Tobita, H.: Cloud Interface: Designing aerial computer environment for novel user interface. In: Proceedings of the 8th International Conference on Advances in Computer Entertainment Technology. pp. 57:1-57:8. ACE '11, ACM, New York, NY, USA (2011), http://doi. acm.org/10.1145/2071423.2071495

16. Tokuda, Y., Suzuki, Y., Nishimura, K., Tanikawa, T., Hirose, M.: Cloud Display. In: Proceedings of the 7th International Conference on Advances in Computer Entertainment Technology. pp. 32-35. ACE '10, ACM (2010), http://doi.acm.org/10.1145/1971630. 1971640

17. Virolainen, A., Puikkonen, A., Kärkkäinen, T., Häkkilä, J.: Cool Interaction with Calm Technologies: Experimenting with ice as a multitouch surface. In: Proceedings of ACM International Conference on Interactive Tabletops and Surfaces. pp. 15-18. ITS '10, ACM (2010), http://doi.acm.org/10.1145/1936652.1936656

18. Yonezawa, T., Mase, K.: Tangible Sound: Musical instrument using fluid water. In: Proceedings of International Computer Music Association. ICMC2000, Citeseer (2000) 